\title{
Robust Control Design for Perturbed Systems by Frequency Domain Approach
}

\author{
Bing-Fei Wu, Senior Member, IEEE, Hung-I Chin, Member, IEEE, Jau-Woei Perng, Member, IEEE, \\ and Tsu-Tian Lee, Fellow, IEEE
}

\begin{abstract}
This paper is concentrated on a perturbed vehicle control system whose gain margin (GM) and phase margin (PM) are analyzed and for which a novel controller design method satisfying the given specifications on GM, PM, and sensitivity is developed. The approach is applied to the plants with uncertain parameters that vary in intervals. Based on the parameter space method and robust stability criteria, gain and phase boundary curves are generated from the characteristic polynomial of the system with which a gain-phase tester is included in series to perform system stability analysis and controller design. The main concern in the controller design is to find a region in the controller coefficient plane so that the performance of the uncertain system satisfies given specifications. The proposed method is applied to an example of a bus system. Simulation results are given for illustration to show the system performances on GM and PM, and the desired controller meeting the specified conditions in frequency domain for the perturbed system is derived.
\end{abstract}

Index Terms-Gain margin (GM) and phase margin (PM), parameter space method, robust control, sensitivity.

\section{INTRODUCTION}

G AIN MARGIN (GM) and phase margin (PM) are important specifications in the frequency domain for the analysis and design of practical control systems and have served as important measures of robustness analysis, which is always of primary concern. This is because the models used are usually imprecise, and the parameters of all physical systems vary with the operating conditions and time. They are usually obtained numerically or graphically by the use of system frequency response like Bode plots. Studying for controller design to satisfy GM, PM, or sensitivity conditions was proposed by several articles, such as in [1]-[6]. There are also many design methods used in determining the parameters to meet different objectives [7]-[9]. Designing a controller for a fixed and exact control plant is not usually practical in the natural environments. Due to the simplified models or the factors resulting from the changing environments, the uncertainties in system parameters can

Manuscript received June 28, 2006; revised December 14, 2006 and December 15, 2006. This work was supported by National Science Council under Grant NSC 95-2752-E-009-012-PAE. The review of this paper was coordinated by Dr. M. Abul Masrur.

B.-F. Wu and J.-W. Perng are with the Department of Electrical and Control Engineering, National Chiao Tung University, Hsinchu 300, Taiwan, R.O.C. (e-mail: bwu@cc.nctu.edu.tw).

H.-I Chin is with the Department of Electronic Engineering, Minghsin University of Science and Technology, Hsinchu 304, Taiwan, R.O.C.

T.-T. Lee is with the Department of Electrical Engineering, National Taipei University of Technology, Taipei 106, Taiwan, R.O.C.

Color versions of one or more of the figures in this paper are available online at http://ieeexplore.ieee.org.

Digital Object Identifier 10.1109/TVT.2007.900485 always occur. Uncertain parameters in a linear control system can be robustly analyzed by the parameter plane method or the parameter space method [10]-[17]. A simple way of checking the stability of perturbed interval polynomials by Kharitonovbased robustness analysis methods is to guarantee if all the polynomials have the roots in the left-half plane [18]. The perturbed parameters will result in root clusters, within which the roots of the perturbed polynomials will be located. Usually, a change in a physical quantity typically appears in more than one coefficient of the characteristic equation. Robust Gammastability analysis for a perturbed vehicle plant was also studied [19]. The methods of analyzing the GM-PM of a linear control system with adjustable parameters have been developed [20][22]. Strictly speaking, the majority of the research mentioned above is not concentrated on the controller design for perturbed systems. Sensitivity functions are usually used as a design specification to indicate the robustness of a system. In [4] and [6], Yaniv and Nagurka proposed a robust controller design method satisfying GM, PM, and sensitivity constraints on the perturbed systems, not with the system parameters in uncertain continuous intervals, but with the system uncertainties in the finite discrete set of gains and pole locations.

In this paper, GM and PM performances are defined for a perturbed system with uncertain continuous interval parameters and shown here graphically in 2-D and 3-D in the system parameter space. By the use of the parameter space method and robustness stability criteria, stability boundary curves corresponding to specific GM and PM constraints are generated. Owing to the complexity of the controller design for perturbed control systems, it is not an easy job to find out a qualified controller together with the system plant with uncertain interval parameters so that the whole closed system at every point in the perturbed system parameter region satisfies all the three specifications of GM, PM, and sensitivity. The main concern in the controller design is to find a controller region in the controller coefficient plane so that the performance of the whole system with uncertain parameters inside a perturbed space satisfies the given specifications. The desired controller will be determined graphically from a figure in which a qualified controller coefficient area is to be found out. With the help of stability boundary curves in the controller coefficient space, the objective of designing a suitable controller meeting the specified requirements is achieved.

This paper is organized as follows. The basic robust stability concept for the topic is formulated in Section II. In Section III, sensitivity functions are to be defined, and constant-sensitivity loci will be generated. Section IV presents stability boundary 


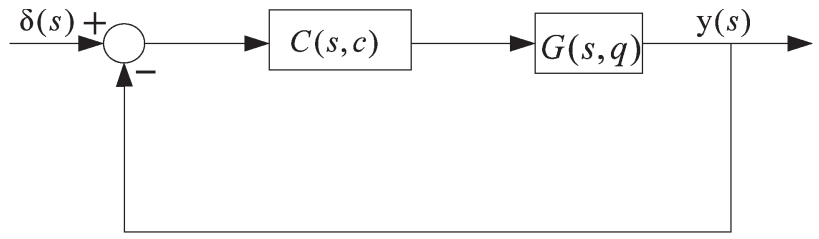

Fig. 1. Perturbed vehicle control system with uncertain parameter $q$.

analysis. GM and PM analysis is described, and the controller design algorithm is proposed. In Section V, a linearized bus model that is taken as an example with perturbed parameters is presented to analyze the GM and PM performances and to design a qualified controller. Simulation results are provided in this section. Finally, conclusions are given in Section VI.

\section{BASIC RoBUSt STABILITY CONCEPT}

Boundary curves are to be constructed to separate the parameter space into stable and unstable regions. At the interior points of a stable region in the parameter space, the roots of the characteristic equation of the closed system lie in the left-half part in the $s$-plane, but the unstable region contains the points at which the system has unstable roots.

Consider a linear control feedback system illustrated in Fig. 1. The closed-loop feedback system has the transfer function given by

$$
H(s, q)=\frac{C(s) G(s, q)}{1+C(s) G(s, q)}
$$

where $C(s)$ is a controller with constant coefficients, and $G(s, q)$ is a plant with a perturbed parameter vector $q=$ $\left[q_{1}, q_{2}, \ldots, q_{n}\right] \in R$. $R$ is a set of allowable parameter domain space. Each $q_{i}$ varies independently within the interval with $q_{i} \in\left[q_{i}^{-} ; q_{i}^{+}\right], i=1,2, \ldots, n$.

The characteristic polynomial is

$$
P(s, q)=\sum_{i=0}^{n} d_{i}(q) s^{i}=d_{0}(q)+d_{1}(q) s+\cdots+d_{n}(q) s^{n} .
$$

It has been shown that, for real continuous coefficient functions $d_{i}(q)$ of the characteristic equation, a necessary and sufficient condition for robust stability is as follows. 1) There exists $q=q_{o} \in R$ such that $P(s, q)$ is stable. 2) $P(s, q)$ does not have any roots on the imaginary axis for any $q \in R$ [19]. It is easily tested by checking the stability of the characteristic polynomial $P\left(s, q_{o}\right)$ for an arbitrary $q_{0} \in R$. If no such $q_{o}$ exists, the system is unstable. The condition 2) is satisfied if and only if the equation $P(s, q)=0$ neither has a real root at $s=0$, i.e.,

$$
d_{0}(q) \neq 0
$$

nor an imaginary pair of roots at $s= \pm j \omega$ for all $q \in R$. Let $R_{j \omega}$ be the set of all real $q$ such that the polynomial $P(s, q)$

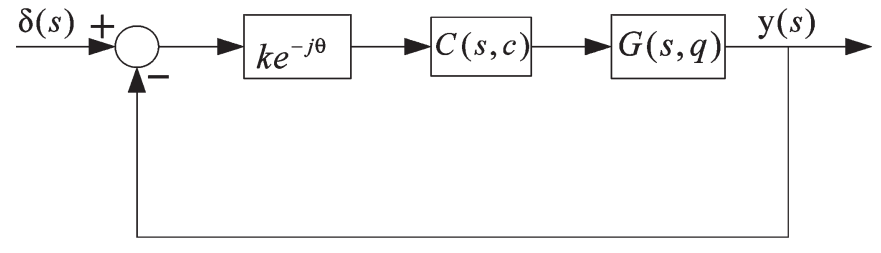

Fig. 2. Perturbed vehicle control system in series with a gain-phase tester $k e^{-j \theta}$.

has roots on the imaginary axis

$$
R_{j \omega}=\{q: P(j \omega, q)=0 \text { for } \omega \geq 0\} .
$$

The condition 2) also means that $R_{j \omega}$ does not intersect the parameter domain space $R$.

The curve formed by the points $q$ in $R_{j \omega}$ in the $q$-space is the stability boundary curve. The perturbed vehicle system is stable at the points in the $q$-space on one side of the stable boundary curve and unstable at the points on the other side.

\section{SEnsitivity}

Since, in physical systems, all the elements may change their properties with time and environment, the considerations about the changes of the characteristics of the closed control systems with respect to system parameter variations are always of big concern for a system designer. Consider the closed control system in (1) in which its controller $C(s)$ is replaced by $C(s, c)$, where $c=\left[c_{0}, c_{1}, \ldots, c_{m}\right]$, and $c_{i}$ is a controller coefficient to be designed for $i=0,1,2, \ldots, m$. The transfer function is given by

$$
H(s, q, c)=\frac{C(s, c) G(s, q)}{1+C(s, c) G(s, q)}
$$

With a specific $q, H(s, q, c)$ is replaced by $H(s, c)$.The sensitivity function $S_{c_{i}}^{H(s, c)}$ with respect to the controller coefficient $c_{i}$ is defined as

$$
S_{c_{i}}^{H(s, c)}=\frac{d H(s, c) / H(s, c)}{d c_{i} / c_{i}}
$$

where $i=0,1,2, \ldots, m$. Given a different constant $s_{0}$, the solutions of the equality $\left|S_{c_{i}}^{H(j \omega)}\right|_{s=j \omega} \mid=s_{0}$ for a controller coefficient $c$ give constant-sensitivity loci in the $c$-space. The controller coefficient $c$ will be determined based on sensitivity specifications corresponding to one of those loci. A system being very insensitive to parameter variations is considered to be a good control system.

\section{STABility BOUndary ANAlysis}

Consider a gain-phase tester $k e^{-j \theta}$ to be included in series with the original control system, as in Fig. 2, and its transfer function is given by

$$
H(s, q, c, K, \theta)=\frac{K e^{-j \theta} C(s, c) G(s, q)}{1+K e^{-j \theta} C(s, c) G(s, q)} .
$$


The closed-loop characteristic polynomial is $P(s, q, c$, $k, \theta)$, and

$$
\begin{aligned}
P(s, q, c, k, \theta)= & \text { the numerator of }\left[1+k e^{-j \theta} C(s, c) G(s, q)\right] \\
= & \sum_{i=0}^{n} d_{i}(q, c, k, \theta) s^{i} \\
= & d_{0}(q, c, k, \theta)+d_{1}(q, c, k, \theta) s \\
& +\cdots+d_{n}(q, c, k, \theta) s^{n} .
\end{aligned}
$$

By the use of the parameter space method and robust stability criteria, system stability performance on GM and PM is analyzed by generating the gain and phase boundary curves. For perturbed control systems in which the parameters of the characteristic polynomial lie within the given intervals, the minimum of all the GM values of the system at the points inside the entire perturbed region in the system parameter plane is defined to be the GM of the system. The PM of the system is defined in the same way.

\section{A. Parameter Space Method}

The parameter space method is a good analytical technique to perform system analysis in the selected system parameter plane for a control system which is described by the characteristic polynomial equation of which the roots generate stability boundary curves in the parameter plane. The characteristic polynomial on the $j \omega$-axis $P(j \omega, q, c, k, \theta)$ may be written into the real part $U(\omega, q, c, k, \theta)$ and the imaginary part $V(\omega, q, c, k, \theta)$

$$
P(\omega, q, c, k, \theta)=U(\omega, q, c, k, \theta)+j V(\omega, q, c, k, \theta)=0
$$

where

$$
\begin{aligned}
U(\omega, q, c, k, \theta) & =r_{0}(q, c, k, \theta)+r_{1}(q, c, k, \theta) \omega \\
+ & r_{2}(q, c, k, \theta) \omega^{2}+\cdots+r_{n}(q, c, k, \theta) \omega^{n}
\end{aligned}
$$

and

$$
\begin{aligned}
V(\omega, q, c, k, \theta) & =i_{0}(q, c, k, \theta)+i_{1}(q, c, k, \theta) \omega \\
+ & i_{2}(q, c, k, \theta) \omega^{2}+\cdots+i_{n}(q, c, k, \theta) \omega^{n} .
\end{aligned}
$$

The equations

$$
\left\{\begin{array}{l}
U(\omega, q, c, k, \theta)=0 \\
V(\omega, q, c, k, \theta)=0
\end{array}\right.
$$

can be solved analytically or numerically for $q$ or $c$. Gain and phase boundary curves are generated both in $q$-space from the solutions $q$ for GM and PM analysis and in $c$-space from the solutions $c$ for the controller design under specified conditions.

In the analysis of GM and PM, a fixed controller is used to analyze the system performance, and (9)-(12) do not depend on $c$. The GM and PM of the perturbed vehicle system will be analyzed geometrically in two and three dimensions from stability boundary curves.
In controller design, (12) can be solved for $c$ with specific $\omega, k, \theta$, and $q$ in a similar way. Gain and phase boundary curves are developed in the $c$-space according to different gain $k$ and $\theta$, respectively.

\section{B. GM Analysis}

Let $\theta=0^{\circ}$ and $c$ be a specific controller coefficient in (12), and solve (12) for $q$. A gain boundary curve is generated in $q$-space from the solutions $q$ by varying $\omega$ for every $k$. By varying $k$, the curve is approaching to the parameter region $R$ gradually and finally intersects with $R$. A specific gain $k$ (in decibels) corresponding to the boundary curve, which is tangent to the perturbed region $R$, is defined as the GM of the perturbed control system. It is also the minimal GM of the system within the entire region $R$. The GM of the control system at a point on one side of a specific gain boundary curve is greater than that at a point on the boundary curve. However, it is less at the points on the other side.

\section{PM Analysis}

Given $k=1$ and a specific $c$, repeat the process as previously. Phase boundary curves are developed under the PM specification in a similar way. They are generated in $q$-space from the solutions $q$ by varying $\omega$ for every $\theta$. The PM of the control system is defined as the phase value $\theta$ associated with the phase boundary curve which is tangent to the perturbed region $R$. It is the minimal PM for the whole system with the parameters inside $R$ too. The PM of the control system at a point on one side of a specific phase boundary curve is greater than that at a point on the boundary curve. However, it is less at a point on the other side.

\section{Controller Design}

The controller design is based on gain-phase boundary curves that are drawn in $c$-space from the locations of the roots of (12) with respect to different $k$ and $\theta$, and the constantsensitivity loci that are drawn based on the solutions of the $\left|S_{c_{i}}^{H(j \omega)}\right|_{s=j \omega} \mid=s_{0}$ for the controller coefficient $c$ in $c$-space with respect to the given sensitivity constant $s_{0}$. The desired coefficients are determined under the constraints of specified GM, PM, and sensitivity. Systems with high stability and low sensitivity are desired.

Based on the discussions mentioned above, the design algorithm is as follows.

Step 1) Set up user-defined specifications on GM, PM, and sensitivity.

Step 2) For every system parameter $q$ at the vertices of the perturbed system parameter region in $q$-plane, draw the gain boundary curves corresponding to the specified GM and $0 \mathrm{~dB}$ in $c$-plane by solving (12).

Step 3) For every $q$ at the vertices of the perturbed system parameter region in $q$-plane, draw the phase boundary curves corresponding to the specified $\mathrm{PM}$ and $0^{\circ}$ in $c$-plane by solving (12). 
Step 4) Sketch the sensitivity constant loci from the solutions of the equality $\left|S_{c_{i}}^{H(j \omega)}\right|_{s=j \omega} \mid=s_{0}$ for $c$, given $s_{0}$.

Step 5) Determine a gain region in $c$-space with the help of the gain boundary curves as in Step 2) so that the controller with the coefficients in that region satisfies the specified GM constraints.

Step 6) Determine a phase region in $c$-space with the help of the phase boundary curves as in Step 3) so that the controller with the coefficients in that region satisfies the specified PM constraints.

Step 7) Find out the common region of the determined gain and phase ones as in Steps 5) and 6). The controller with the coefficients in that region is the desired one satisfying the specified GM and PM conditions.

Step 8) Choose a point in $c$-space on a specified sensitivity constant locus which passes through the common region as in Step 7). Then, the controller coefficient at that point satisfies all the three specified constraints of GM, PM, and sensitivity. If no such sensitivity locus exists, tradeoff has to be made among the three specified conditions.

\section{EXAMPle AND Simulation Results}

In this simulation, a Daimler Benz 0305 bus [19] is adopted. Its linearized system, with actuator input $\delta=$ steering angle rate, and output $y=$ displacement of front antenna, has the following transfer function:

$G\left(s, q_{1}, q_{2}\right)=\frac{609.8 q_{1}^{2} q_{2} s^{2}+388600 q_{1} s+48280 q_{1}^{2}}{s^{3}\left(q_{1}^{2} q_{2}^{2} s^{2}+1077 q_{1} q_{2} s+16.8 q_{1} q_{2}+270000\right)}$

where the parameter $q_{1}=\nu$ is the bus velocity, and the other parameter $q_{2}=m / u$, where $m$ is the mass of the bus (tons), and $u$ is the road friction coefficient ( 0.5 for a wet road and 1 for a dry road).

$$
\begin{aligned}
& q_{1} \in\left[12 \mathrm{~m} \cdot \mathrm{s}^{-1}, \quad 20 \mathrm{~m} \cdot \mathrm{s}^{-1}\right] \\
& q_{2} \in[24 \text { tons, } \quad 32 \text { tons }] .
\end{aligned}
$$

\section{A. GM and PM Analysis}

The controller used is taken as given by

$$
C(s)=\frac{2344 s^{2}+10938 s+9375}{s^{3}+50 s^{2}+1250 s+15625}
$$

and was determined by Muench [19].

Case 1-2-D GM/PM Analysis in $q_{1}-q_{2}$ Plane: Consider the system parameter $q=\left[q_{1}, q_{2}\right]$ with an uncertain parameter region $R$, as in Fig. 3, for studying GM/PM performances.

The $R$-parameter region is given as

$$
\left\{\begin{array}{l}
12 \leq q_{1} \leq 20 \\
24 \leq q_{2} \leq 32
\end{array}\right.
$$

and the closed-loop characteristic polynomial is given as in (8). By substituting $s=j \omega$ into the numerator of the above

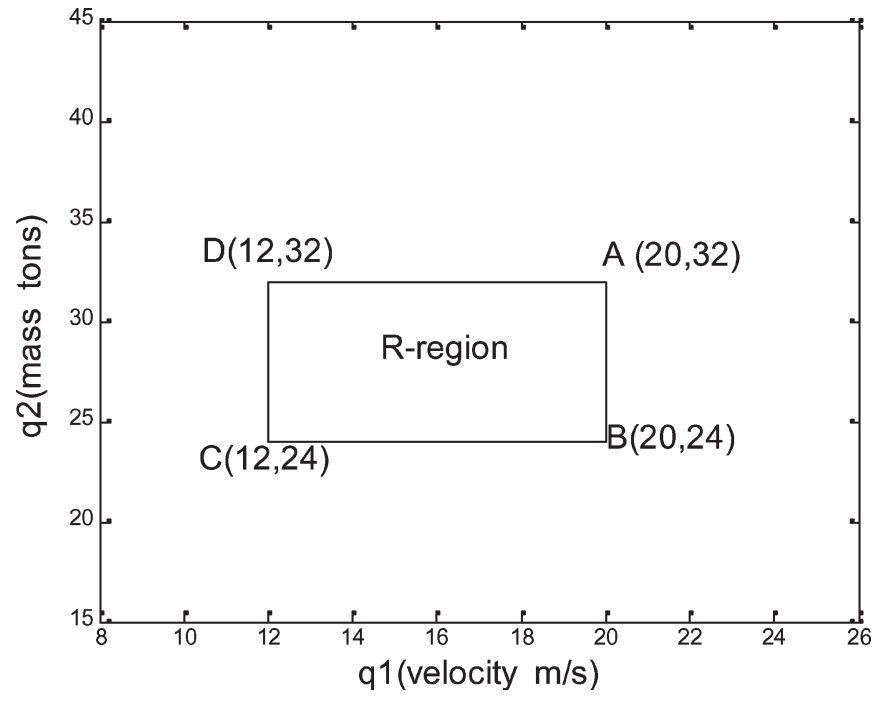

Fig. 3. Parameter domain region in $q 1-q 2$ plane.

polynomials and by lengthy computation, the coefficients of the real-part polynomial $U(\omega, q, k, \theta)$ with a specific $c$ in (10) are

$$
\begin{aligned}
r_{0}= & 4.5262 \times 10^{8} q_{1}^{2} k \cos (\theta) \\
r_{1}= & \left(3.6431 \times 10^{9} q_{1}+5.2808 \times 10^{8} q_{1}^{2}\right) \times k \sin (\theta) \\
r_{2}= & \left(-4.2505 \times 10^{9} q_{1}-5716875 q_{1}^{2} q_{2}-1.1316 \times 10^{8} q_{1}^{2}\right) \\
& \times k \cos (\theta) \\
r_{3}= & -\left(6669992.4 q_{1}^{2} q_{2}+9.1087 \times 10^{8} q_{1}\right) k \sin (\theta) \\
r_{4}= & 16828125 q_{1} q_{2}+21000 q_{1}^{2} q_{2}+3375 \times 10^{5} \\
& +1429371.2 k q_{1}^{2} q_{2} \cos (\theta) \\
r_{5}= & 0 \\
r_{6}= & -1250 q_{1}^{2} q_{2}^{2}-16.8 q_{1}^{2} q_{2}-53850 q_{1} q_{2}-270000 \\
r_{7}= & 0 \\
r_{8}= & q_{1}^{2} q_{2}^{2} .
\end{aligned}
$$

In (11), the coefficients of the imaginary-part polynomial $V(\omega, q, k, \theta)$ are

$$
\begin{aligned}
i_{0}= & -4.5262 \times 10^{8} k q_{1}^{2} \sin (\theta) \\
i_{1}= & \left(3.6431 \times 10^{9} q_{1}+5.2808 \times 10^{8} k q_{1}^{2} \cos (\theta)\right. \\
i_{2}= & \left(4.2505 \times 10^{9} q_{1}+5716875 q_{1}^{2} q_{2}+1.1316 \times 10^{8} q_{1}^{2}\right) \\
& \times k \sin (\theta) \\
i_{3}= & -262500 q_{1}^{2} q_{2}-4218750000-6669992.4 \\
& \times k q_{1}^{2} q_{2} k \cos (\theta)-9.1087 \times 10^{8} k q_{1} \cos (\theta) \\
i_{4}= & -1429371.2 k q_{1}^{2} q_{2} \sin (\theta) \\
i_{5}= & 15625 q_{1}^{2} q_{2}^{2}+840 q_{1}^{2} q_{2}+1346250 q_{1} q_{2}+135 \times 10^{5} \\
i_{6}= & 0 \\
i_{7}= & -50 q_{1}^{2} q_{2}^{2}-1077 q_{1} q_{2} .
\end{aligned}
$$

We solve (12) for $q$ by varying $k$ and $\theta$, and the stable boundary representation curves for GM and PM are shown in Figs. 4 and 5 in the $q_{1}-q_{2}$ plane, respectively. We are only interested in positive solutions $q_{1}>0$ and $q_{2}>0$ for practical reasons. The 


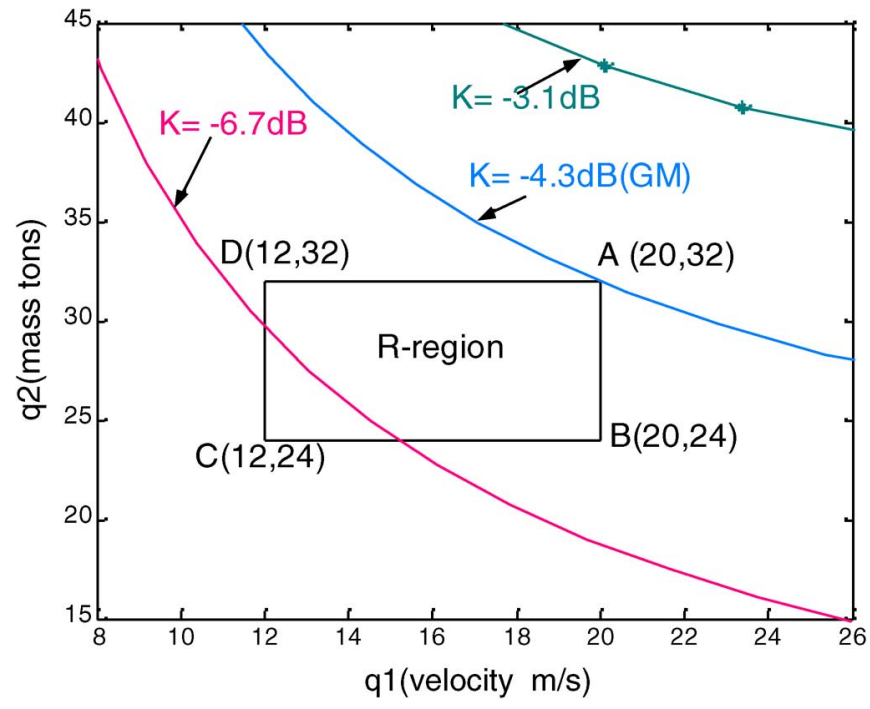

Fig. 4. Gain boundary curves by varying $k$ with $\mathrm{GM}=-4.3 \mathrm{~dB}$.

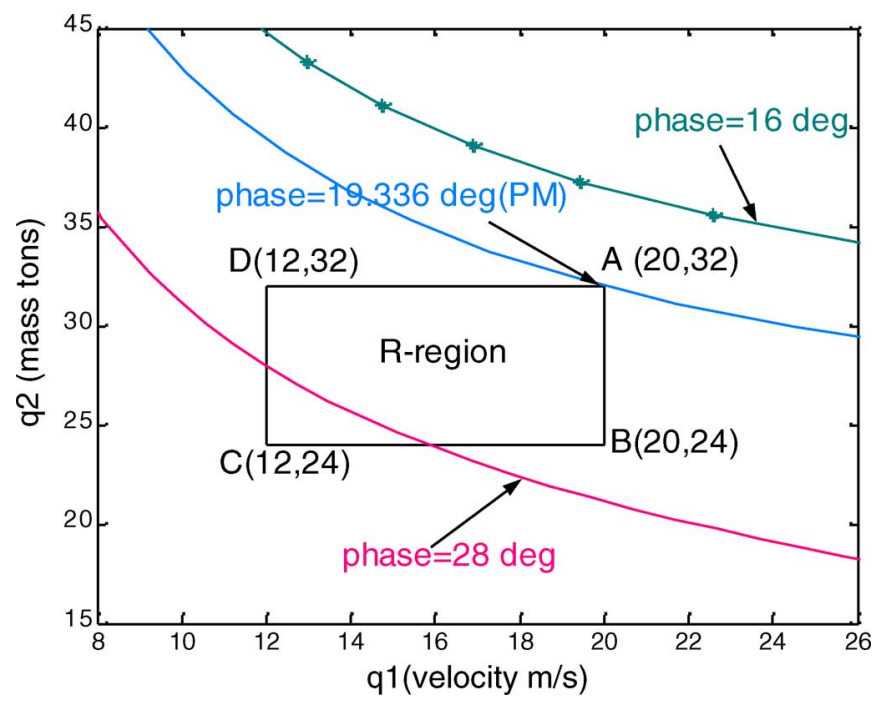

Fig. 5. Phase boundary curves by varying $\theta$ with $\mathrm{PM}=19.336^{\circ}$.

GM of the perturbed control system with the domain region $R$ is $-4.3 \mathrm{~dB}$, and its $\mathrm{PM}$ is $19.336^{\circ}$, as shown in Figs. 4 and 5, respectively. In general, the specifications on the stability robustness point of view are $\mathrm{GM} \geq 3 \mathrm{~dB}$ and $\mathrm{PM} \geq 30^{\circ}$, which the system with the original controller (15) does not satisfy. A new controller is designed in the following section, and its performance is improved significantly.

The gain boundary curves associated with different gains, as shown in Fig. 4, reveal that the GM of the control system at a point on one side of a specific gain boundary curve is greater than that at a point on the curve. However, it is less at a point on the other side.

Similarly in Fig. 5, the phase boundary curves show that the $\mathrm{PM}$ of the control system at a point on one side of a specific phase boundary curve is greater than that at a point on the curve. However, it is less at a point on the other side. At the point $\mathrm{A}\left(\left(q_{1}, q_{2}\right)=(20,32)\right)$ in both figures, the system has the minimal GM and PM of all the points within the entire $R$ region.

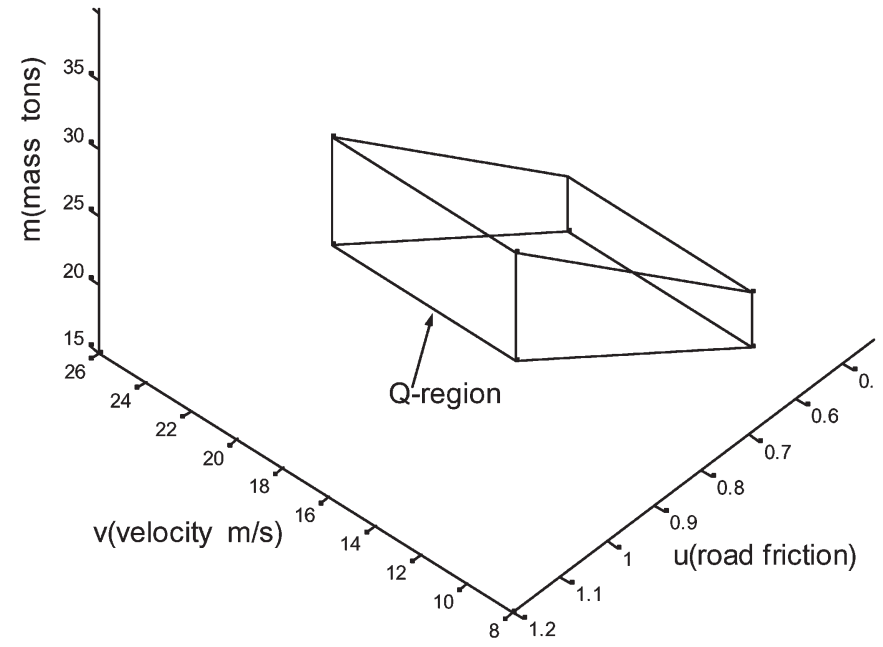

Fig. 6. Three-dimensional perturbed parameter space $Q$ with three uncertain parameters $m, \nu$, and $u$.

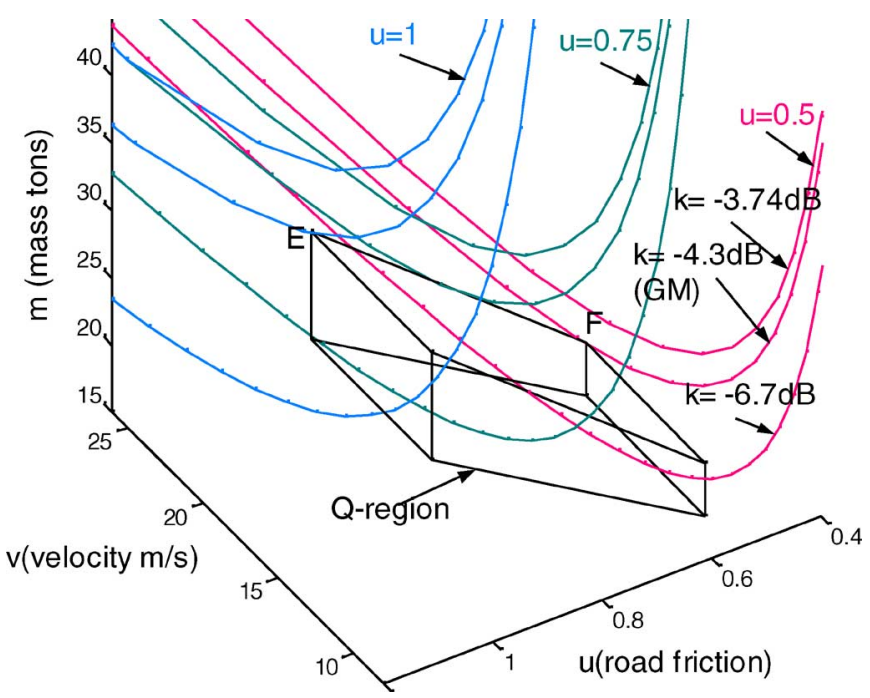

Fig. 7. Gain boundary curves in 3-D by varying $k$ with $\mathrm{GM}=-4.3 \mathrm{~dB}$.

Case 2-3-D GM/PM Analysis in $m-\nu-u$ Space: Select $q=\left[q_{1}, q_{2}, q_{3}\right]=[m, \nu, u]$ in the block diagram of the closed system in Fig. 2. The perturbed parameter space $Q$, as in Fig. 6, is as follows:

$$
\left\{\begin{array}{l}
24 \leq m / u \leq 32 \\
12 \leq \nu \leq 20 \\
0.5 \leq u \leq 1
\end{array}\right.
$$

The gain and phase boundary curves in the $m-\nu-u$ parameter space are generated from the solutions for $q$ to (10). Those curves corresponding to different $k$ and $\theta$ by varying the frequency $\omega$ are shown in Figs. 7 and 8. A specific gain $k$ (in decibels) corresponding to a boundary curve, which is tangent to the perturbed region $Q$ at a point on the edge $\overline{\mathrm{EF}}$ of $Q$, is defined as the GM of the system. It is also the minimal GM of the perturbed control system within $Q$. Its PM is defined in the same way. The system with uncertain parameters within the $Q$-space has $\mathrm{GM}=-4.3 \mathrm{~dB}$ and $\mathrm{PM}=19.336^{\circ}$. 


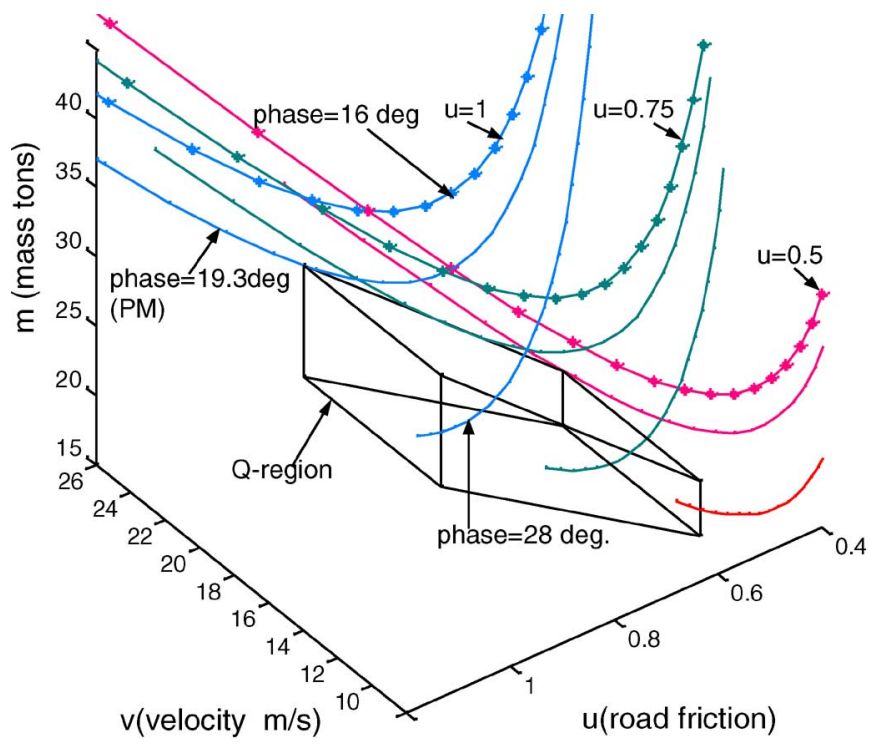

Fig. 8. Phase boundary curves in 3-D with $\mathrm{PM}=19.336^{\circ}$.

\section{B. Controller Design}

The system parameter $q=\left[q_{1}, q_{2}\right]$ within $R$ is considered for the controller design.

Assume the controller to be designed is given as

$$
C(s)=\frac{c_{2} s^{2}+c_{1} s+c_{0}}{s^{3}+50 s^{2}+1250 s+15625}
$$

where $c_{0}, c_{1}$, and $c_{2}$ are the controller coefficients to be designed under the user-specified constraints, and the system parameter domain is within the region $R$, as in Fig. 3. Equation (15) is a special case of (20) with $c_{0}=9375, c_{1}=10938$, and $c_{2}=2344$.

Specification 1-A Controller Design for $G M \geq 3 \mathrm{~dB}$ and $P M \geq 30^{\circ}$ : The design problem of interest is to find all the controller coefficients $c_{0}, c_{1}$, and $c_{2}$ that satisfy the userspecified conditions of GM and PM. According to the design steps, a coefficient region in $c$-space is to be found out by the use of gain and phase boundary curves that are associated with different $k$ and $\theta$.

By solving (12), the coefficients of the real part of the characteristic polynomial $U(\omega, q, c, k, \theta)$ in (10) are

$$
\begin{aligned}
r_{0}= & 48280 c_{0} q_{1}^{2} k \cos (\theta) \\
r_{1}= & \left(388600 c_{0} q_{1}+48280 c_{1} q_{1}^{2}\right) \times k \sin (\theta) \\
r_{2}= & -\left(388600 c_{1} q_{1}+609.8 c_{0} q_{1}^{2} q_{2}+48280 c_{2} q_{1}^{2}\right) k \cos (\theta) \\
r_{3}= & -\left(609.8 c_{1} q_{1}^{2} q_{2}+388600 c_{2} q_{1}\right) k \sin (\theta) \\
r_{4}= & 16828125 q_{1} q_{2}+21000 q_{1}^{2} q_{2} \\
& +3375 \times 10^{5}+609.8 q_{1}^{2} q_{2} c_{2} k \cos (\theta) \\
r_{5}= & 0 \\
r_{6}= & -1250 q_{1}^{2} q_{2}^{2}-16.8 q_{1}^{2} q_{2}-53850 q_{1} q_{2}-270000 \\
r_{7}= & 0 \\
r_{8}= & q_{1}^{2} q_{2}^{2} .
\end{aligned}
$$

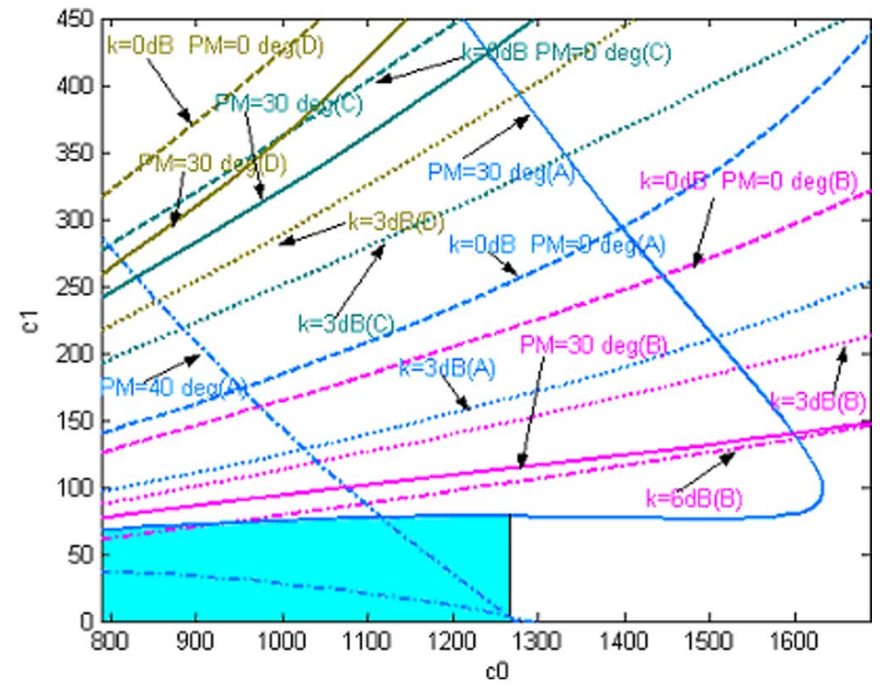

Fig. 9. Controller coefficient region for $\mathrm{GM} \geq 3 \mathrm{~dB}$ and $\mathrm{PM} \geq 30^{\circ}$, as indicated in the shaded area in $c_{0}-c_{1}$ plane with $c_{2}=2344$.

The coefficients of the imaginary part of the polynomial $V(\omega, q, c, k, \theta)$ in (11) are

$$
\begin{aligned}
i_{0}= & -48280 c_{0} q_{1}^{2} k \sin (\theta) \\
i_{1}= & \left(388600 c_{0} q_{1}+48280 c_{1} q_{1}^{2}\right) k \cos (\theta) \\
i_{2}= & \left(388600 c_{1} q_{1}+609.8 c_{0} q_{1}^{2} q_{2}+48280 c_{2} q_{1}^{2}\right) k \sin (\theta) \\
i_{3}= & -262500 q_{1}^{2} q_{2}-4218750000-609.8 c_{1} q_{1}^{2} q_{2} k \cos (\theta) \\
& -388600 c_{2} q_{1} k \cos (\theta) \\
i_{4}= & -609.8 c_{2} q_{1}^{2} q_{2} k \sin (\theta) \\
i_{5}= & 15625 q_{1}^{2} q_{2}^{2}+840 q_{1}^{2} q_{2}+1346250 q_{1} q_{2}+135 \times 10^{5} \\
i_{6}= & 0 \\
i_{7}= & -50 q_{1}^{2} q_{2}^{2}-1077 q_{1} q_{2} \\
i_{8}= & 0
\end{aligned}
$$

where $q=\left(q_{1}, q_{2}\right)$ is a specific point within $R$, and we solve (12) for $c$.

Two controller coefficients of $c_{0}, c_{1}$, and $c_{2}$ are chosen as adjustable parameters, and the other one is fixed for this design. A shaded area is determined by gain and phase boundary curves from the solutions for $\left(c_{0}, c_{1}\right)$ pairs with $c_{2}=2344$ under GM and PM specifications given as above in the $c_{0}-c_{1}$ plane, as shown in Fig. 9.

For the vertices A, B, C, and D of $R$, as in Fig. 3, the stability boundary curves are plotted to determine the qualified shaded area. Two gain boundary curves are obtained that are associated with $k=0 \mathrm{~dB}$ and $3 \mathrm{~dB}$, given $\theta=0^{\circ}$ for each vertex. In a similar way, two phase boundary ones are also generated corresponding to $\theta=0^{\circ}$ and $\theta=30^{\circ}$ with $k=1$.

Let $c_{0}=9375$. Select $c_{1}$ and $c_{2}$ as adjustable coefficients. Gain and phase stability curves are generated in the same way in $c_{1}-c_{2}$ plane, and the shaded region within which $c_{1}$ and $c_{2}$ satisfy the specified constraints is found, as shown in Fig. 10.

In Figs. 9 and 10, the desired controller coefficients can be chosen according to the specified GM, PM, and sensitivity 


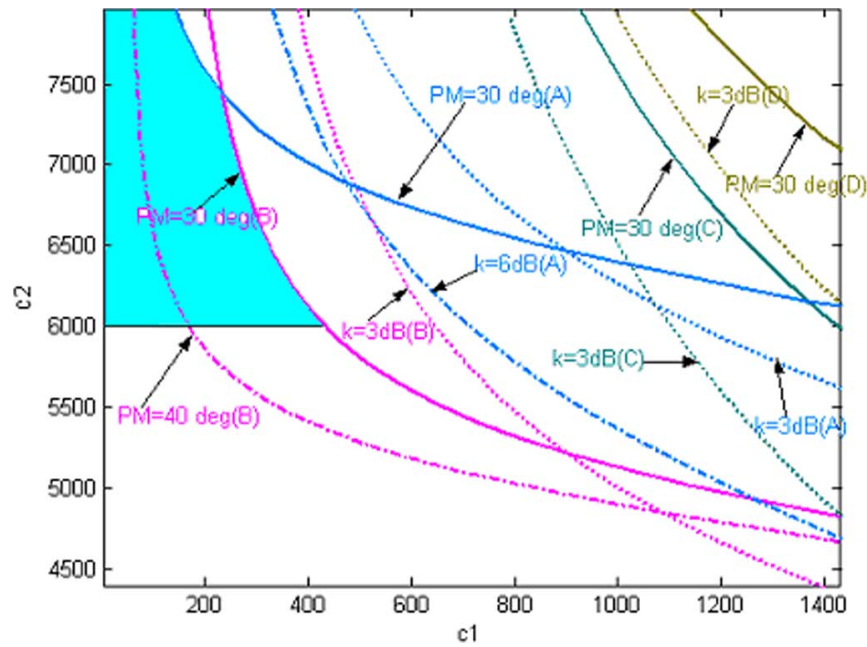

Fig. 10. Controller coefficient region for $\mathrm{GM} \geq 3 \mathrm{~dB}$ and $\mathrm{PM} \geq 30^{\circ}$, as indicated in the shaded area in $c_{1}-c_{2}$ plane with $c_{0}=9375$.

TABLE I

GM AND PM OF THE SYSTEM WITH $c_{2}=2344$

\begin{tabular}{|c|c|c|}
\hline \multicolumn{3}{|c|}{$c_{0}=180.7, c_{1}=18.83$} \\
\hline Location inside R & GM & PM \\
\hline Point A(20,32) & $4.13 \mathrm{~dB}$ & $37.1^{\circ}$ \\
\hline Point B(20,24) & $3.18 \mathrm{~dB}$ & $35^{\circ}$ \\
\hline Point C $(12,24)$ & $9.76 \mathrm{~dB}$ & $67.1^{\circ}$ \\
\hline Point D(12,32) & $10.3 \mathrm{~dB}$ & $64.5^{\circ}$ \\
\hline Point $(17,30)$ & $5.73 \mathrm{~dB}$ & $46.5^{\circ}$ \\
\hline
\end{tabular}

TABLE II

GM AND PM OF THE SYSTEM WiTH $c_{0}=9375$

\begin{tabular}{|c|c|c|}
\hline \multicolumn{3}{|c|}{$c_{1}=410, c_{2}=6000$} \\
\hline Location inside R & GM & PM \\
\hline Point A(20,32) & $8.48 \mathrm{~dB}$ & $57^{\circ}$ \\
\hline Point B(20,24) & $6.08 \mathrm{~dB}$ & $31^{\circ}$ \\
\hline Point $\mathrm{C}(12,24)$ & $6.29 \mathrm{~dB}$ & $55.7^{\circ}$ \\
\hline Point $\mathrm{D}(12,32)$ & $8.64 \mathrm{~dB}$ & $67.7^{\circ}$ \\
\hline Point $(14,26)$ & $6.87 \mathrm{~dB}$ & $57.9^{\circ}$ \\
\hline
\end{tabular}

constraints. The controller coefficient is selected from the above shaded region so that the whole system has the desired stability and sensitivity. With the designed controller, Tables I and II show the GM and PM of the system operating at several points within the region $R$. The Bode plots of magnitude and phase are provided in Figs. 11 and 12.
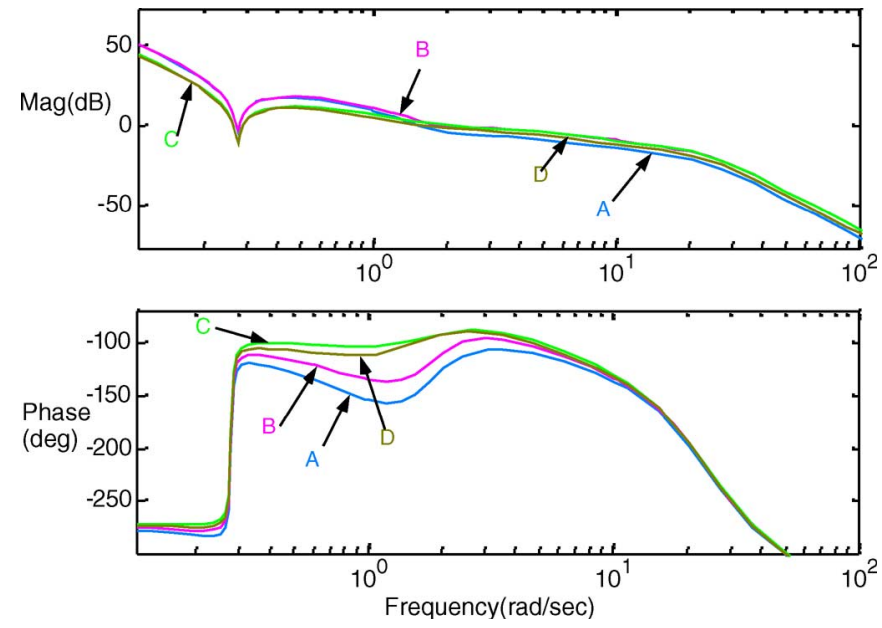

Fig. 11. Bode plots of magnitude and phase with $c_{0}=180.7, c_{1}=18.83$, and $c_{2}=2344$ at four vertices of the perturbed region $R$.
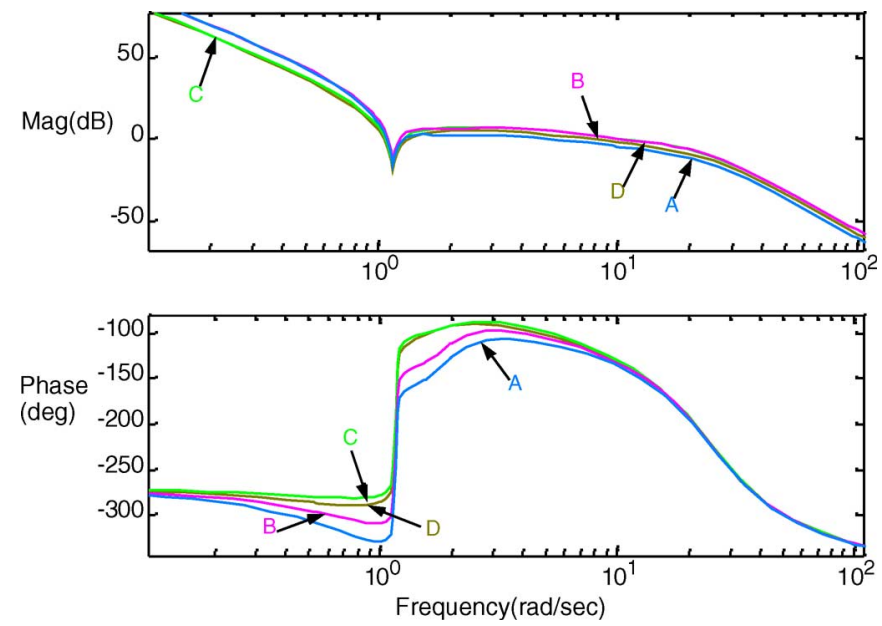

Fig. 12. Bode plots of magnitude and phase with $c_{0}=9375, c_{1}=410$, and $c_{2}=6000$ at four vertices of the perturbed region $R$.

Specification 2-Constant-Sensitivity Loci: Compute $S_{c_{i}}^{H(s, c)}$ for $i=0,1,2$ by substituting (5) and (20) into (6) as follows:

$$
\begin{aligned}
S_{c_{0}}^{H(s, c)} & =\frac{c_{0} D_{c}(s) D_{G}(s)}{N_{c}(s, c)\left(D_{c}(s) D_{G}(s)+N_{c}(s, c) N_{G}(s)\right)} \\
s_{c_{1}}^{H(s, c)} & =\frac{s c_{1} D_{c}(s) D_{G}(s)}{N_{c}(s, c)\left(D_{c}(s) D_{G}(s)+N_{c}(s, c) N_{G}(s)\right)}
\end{aligned}
$$

and

$$
s_{c_{2}}^{H(s, c)}=\frac{s^{2} c_{2} D_{c}(s) D_{G}(s)}{N_{c}(s, c)\left(D_{c}(s) D_{G}(s)+N_{c}(s, c) N_{G}(s)\right)} .
$$

Let $c_{2}=2344$. The constant-sensitivity loci in Fig. 13 are plotted in $c_{0}-c_{1}$ plane from the solutions to the equality $\left|S_{c_{i}}^{H(j \omega)}\right|_{s=j \omega} \mid=s_{01}$, where $s_{01}$ is a specified sensitivity constant, and $i=0,1$. Gain and phase boundary curves in Fig. 11 are plotted with the system operating at the point $\mathrm{A}$ in the region $R$. If the specified sensitivity locus passes through the shaded area, as in Fig. 9, a point on the locus is chosen, and the controller at this location in $c_{0}-c_{1}$ plane is desired. 


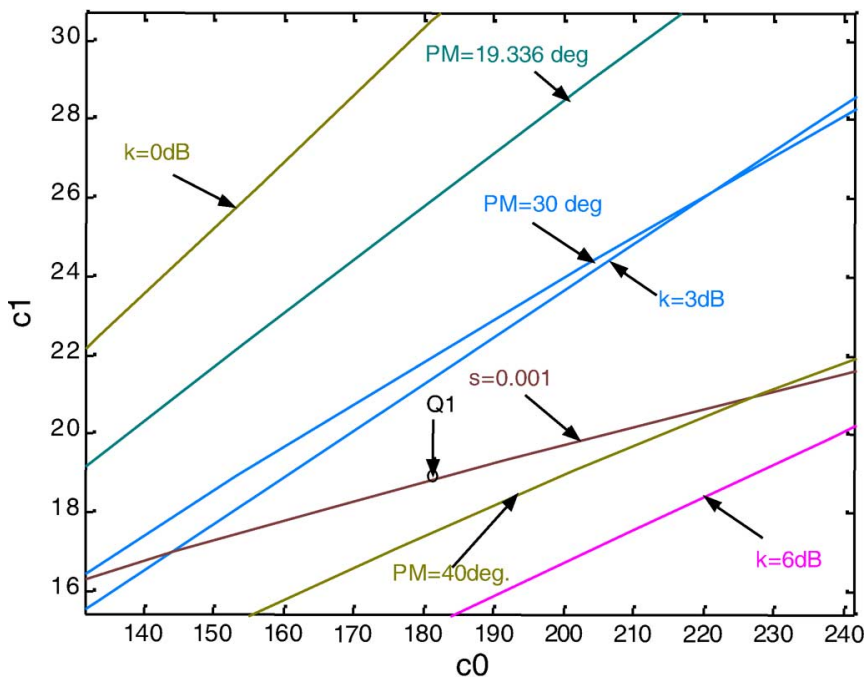

Fig. 13. Chosen controller at the point $Q_{1}$ with $c_{0}=180.7, c_{1}=18.83$, and $c_{2}=2344$ based on the control system at the vertex A $(20,32)$ of the perturbed parameter region $R$

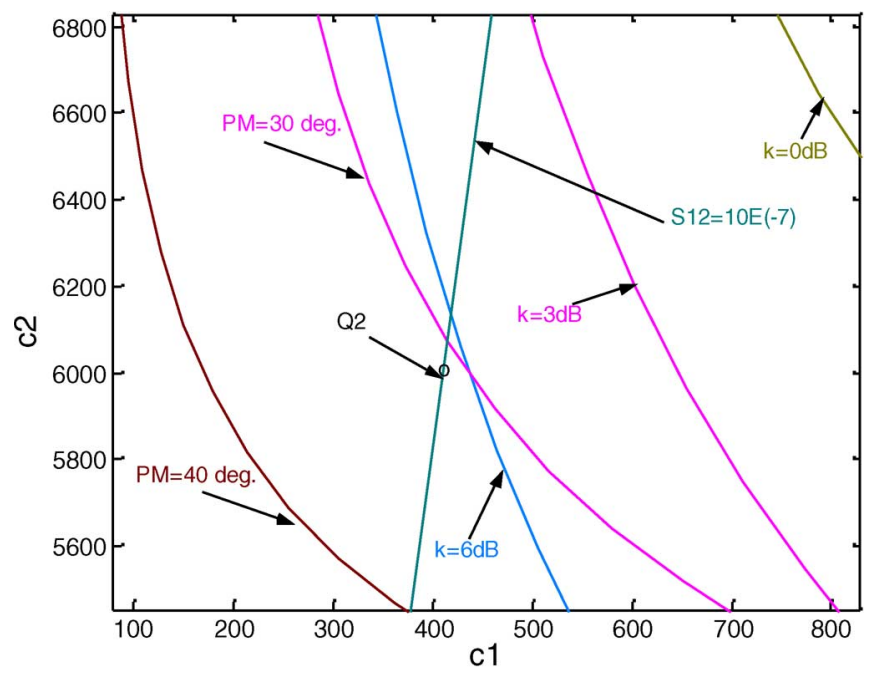

Fig. 14. Chosen controller at the point $Q_{2}$ with $c_{0}=9375, c_{1}=410$, and $c_{2}=6000$ based on the control system at the vertex B $(20,24)$ of the perturbed parameter region $R$.

The point $Q_{1}$ on the sensitivity locus with the constraint $\left.\left|S_{c_{0}}^{H(j \omega)}\right|_{s=j \omega}|=| S_{c_{1}}^{H(j \omega)}\right|_{s=j \omega} \mid=0.001$ is chosen for the controller with $c_{0}=180.7, c_{1}=18.83$, and $c_{2}=2344$. The system at the point $\mathrm{A}$ in $R$ has $\mathrm{GM}=4.13 \mathrm{~dB}$ and $\mathrm{PM}=37.1^{\circ}$. Its performance on stability has been improved.

Let $c_{0}=9375$. The solutions to the equality $\left|S_{c_{i}}^{H(j \omega)}\right|_{s=j \omega} \mid=s_{12}$, where $i=1,2$, give a plot of the constant-sensitivity loci in the $c_{1}-c_{2}$ plane, as shown in Fig. 14. Choose the point $Q_{2}$ in Fig. 14 with $c_{1}=410$ and $c_{2}=6000$ on the sensitivity locus $\left.\left|S_{c_{1}}^{H(j \omega)}\right|_{s=j \omega}|=| S_{c_{2}}^{H(j \omega)}\right|_{s=j \omega} \mid=10^{-7}$, and the system operating at the point $\mathrm{B}$ in $R$ has $\mathrm{GM}=6.08 \mathrm{~dB}$ and $\mathrm{PM}=31^{\circ}$.

\section{CONCLUSION}

This paper introduces a new method of performance analysis and controller design by a frequency-domain approach for a perturbed control system. Based on the parameter space method and robust stability criteria, the performances of a perturbed vehicle control system are analyzed in graphical portrayals and shown in 2-D and 3-D plots. With the help of gain and phase boundary curves resulting from the roots of the system characteristic polynomial equation, the GM and PM have been obtained. In controller design, a methodology is proposed for portraying regions in a selected controller coefficient plane so that the designed controller meets the specified requirements on GM, PM, and sensitivity. Simulation results demonstrate that the objectives have been achieved as desired.

\section{REFERENCES}

[1] C. H. Lee and C. C. Teng, "Tuning of PID controllers for stable and unstable process with specifications on gain and phase margins," Int. J. Fuzzy Syst., vol. 3, no. 1, pp. 346-355, 2001.

[2] G. F. Franklin, J. D. Powell, and A. E. Baeini, Feedback Control of Dynamic Systems. New York: Addison-Wesley, 1986.

[3] K. J. Astrom and T. Hagglund, "Automatic tuning of simple regulators with specifications on phase and amplitude margins," Automata, vol. 20, no. 5, pp. 645-651, 1984.

[4] M. Nagurka and O. Yaniv, "Robust PI controller design satisfying gain and phase margin constraints," in Proc. Amer. Control Conf., 2003, pp. 3931-3936.

[5] W. K. Ho, C. C. Hang, and L. S. Cao, "Tuning of PID controllers based on gain and phase margin specifications," Automatica, vol. 31, no. 3, pp. 497-502, 1995.

[6] O. Yaniv and M. Nagurka, "Robust PI controller design satisfying sensitivity and uncertainty specifications," IEEE Trans. Autom. Control, vol. 48, no. 11, pp. 2069-2072, Nov. 2003.

[7] T. Chai and G. Zhang, "A new self-tuning of PID regulators based on phase and amplitude margin specifications," ACTA Autom. Sin., vol. 23, no. 2, pp. 167-172, 1997.

[8] W. K. Ho, C. C. Hang, and J. H. Zhou, "Self-tuning PID control of a plant with underdamped response with specifications on gain and phase margins," IEEE Trans. Control Syst. Technol., vol. 5, no. 4, pp. 446-452, Jul. 1997.

[9] W. K. Ho and W. Xu, "PID tuning for unstable processes based on gain and phase margin specifications," Proc. Inst. Electr. Eng.-Control Theory Appl., vol. 145, no. 5, pp. 392-396, Sep. 1998.

[10] G. L. Chao, J. W. Perng, and K. W. Han, "Robust stability analysis of time delay systems using parameter-plane and parameter-space method," J. Franklin Inst., vol. 335B, no. 7, pp. 1249-1262, 1998.

[11] S. P. Bhattacharyya, H. Chapellat, and L. H. Keel, Robust Control (The Parametric Approach). Englewood Cliffs, NJ: Prentice-Hall, 1994.

[12] A. Cavallo, G. E. Maria, and L. Verde, "Robust control systems: A parameter space design," J. Guid. Control Dyn., vol. 15, no. 5, pp. 1207-1215, 1992.

[13] D. D. Siljak, "Parameter space methods for robust control design: A guide tour," IEEE Trans. Autom. Control, vol. 34, no. 7, pp. 674-688, Jul. 1989.

[14] D. D. Siljak, "Analysis and synthesis of feedback control systems in the parameter plane," IEEE Trans. Appl. Ind., vol. 83, no. 75, pp. 449-458, Nov. 1964.

[15] J. Ackermann, "Parameter space design of robust control system," IEEE Trans. Autom. Control, vol. AC-25, no. 6, pp. 1058-1072, Dec. 1980.

[16] K. W. Han and G. J. Thaler, "Control system analysis and design using a parameter space method," IEEE Trans. Autom. Control, vol. AC-11, no. 3, pp. 560-563, Jul. 1966.

[17] P. Putz and M. J. Wozny, "A new computer graphics approach to parameter space design of control systems," IEEE Trans. Autom. Control, vol. AC-32, no. 4, pp. 294-302, Apr. 1987.

[18] R. A. Frazer and W. J. Duncan, "On the criteria for the stability of small motions," Proc. R. Soc. Lond. A, Math. Phys. Sci., vol. 124, no. 795, pp. 642-654, Jul. 1929.

[19] J. Ackermann, D. Kaesbauer, and R. Muench, "Robust gamma-stability analysis in a plant parameter space," Automatica, vol. 27, no. 1, pp. 75-85, 1991 .

[20] A. T. Shenton and Z. Shafiei, "Relative stability for control systems with adjustable parameters," J. Guid. Control Dyn., vol. 17, no. 2, pp. 304-310, 1994. 
[21] B. F. Wu, H. I. Chin, and J. W. Perng, "Gain-phase margin analysis of perturbed vehicle control systems," in Proc. IEEE Conf. Netw., Sens. Control, Taipei, Taiwan, R.O.C., 2004, pp. 589-594.

[22] C. H. Chang and K. W. Han, "Gain margins and phase margins for control systems with adjustable parameters," J. Guid. Control Dyn., vol. 13, no. 3, pp. 404-408, 1990.

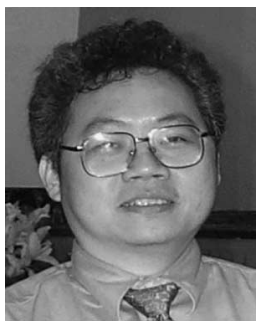

Bing-Fei Wu (S'89-M'92-SM'02) was born in Taipei, Taiwan, R.O.C., in 1959. He received the B.S. and M.S. degrees in control engineering from the National Chiao Tung University (NCTU), Hsinchu, Taiwan, in 1981 and 1983, respectively, and the $\mathrm{Ph} . \mathrm{D}$. degree in electrical engineering from the University of Southern California, Los Angeles, in 1992.

Since 1992, he has been with the Department of Electrical and Control Engineering, NCTU, where he is currently a Professor. He has been involved in the research of intelligent transportation systems for many years and leads a team in developing the first Taiwan smart car (TAIWAN $i$ TS-1) with autonomous driving and active safety system. His current research interests include vision-based vehicle driving safety, intelligent vehicle control, multimedia signal analysis, embedded systems, and chip design.

Prof. Wu founded and served as the Chair of the IEEE Systems, Man, and Cybernetics Society Taipei Chapter in Taiwan in 2003. He has also been the Chair of the Technical Committee of Intelligent Transportation Systems of the IEEE Systems, Man, and Cybernetics Society since 2006. He was the Director of The Research Group of Control Technology of Consumer Electronics in the Automatic Control Section of the National Science Council (NSC), Taiwan, from 1999 to 2000. As an active Industry Consultant, he is also involved with chip design and applications of the flash memory controller and $3 \mathrm{C}$ consumer electronics in multimedia systems and his research on this has been honored by the Ministry of Education with the Best Industry-Academics Cooperation Research Award in 2003. He received the Distinguished Engineering Professor Award from Chinese Institute of Engineers in 2002; the Outstanding Information Technology Elite Award from Taiwan Government in 2003; the Golden Linux Award in 2004; the First Prize Award of TI China-Taiwan DSP Design Contest in 2006; the Outstanding Research Award in 2004 from NCTU; the Research Awards from the NSC in 1992, 1994, and 1996-2000; the Golden Acer Dragon Thesis Award sponsored by the Acer Foundation in 1998 and 2003, respectively; the First Prize Award of the We Win (Win by Entrepreneurship and Work with Innovation and Networking) Competition hosted by the Industrial Bank of Taiwan in 2003; and the Silver Award of Technology Innovation Competition sponsored by the Advantech Foundation in 2003 .

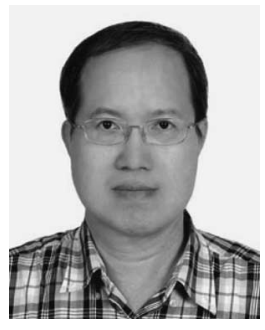

Hung-I Chin (M'06) was born in Taiwan, R.O.C., in 1952. He received the B.S. degree in control engineering from the National Chiao Tung University (NCTU), Hsinchu, Taiwan, in 1976, the M.S. degree in electrical and computer engineering from the State University of New York at Buffalo, in 1986 and the $\mathrm{Ph} . \mathrm{D}$. degree in electrical and control engineering from NCTU in 2005

Since February 1987, he has been with the Department of Electronic Engineering, Minghsin University of Science and Technology, Hsinchu, where he is currently an Associate Professor. His current research interests are robust control systems and fuzzy adaptive systems.

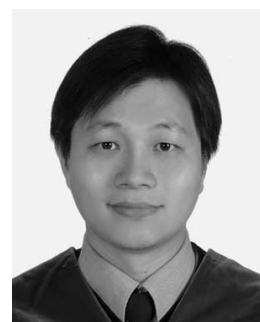

Jau-Woei Perng (S'02-M'04) was born in Hsinchu, Taiwan, R.O.C., in 1973. He received the B.S. and M.S. degrees in electrical engineering from the Yuan Ze University, Chungli, Taiwan, in 1995 and 1997, respectively, and the Ph.D. degree in electrical and control engineering from the National Chiao Tung University (NCTU), Hsinchu, in 2003.

Since 2003, he has been with the Department of Electrical and Control Engineering, NCTU, where he is currently a Research Assistant Professor. His research interests include robust control, nonlinear control, fuzzy logic control, neural networks, systems engineering, and intelligent vehicle control.

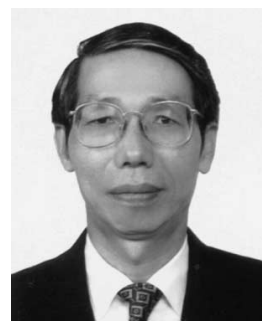

Tsu-Tian Lee (M'87-SM'89-F'97) was born in Taipei, Taiwan, R.O.C., in 1949. He received the B.S. degree in control engineering from the National Chiao-Tung University (NCTU), Hsinchu, Taiwan, in 1970 and the M.S. and Ph.D. degrees in electrical engineering from the University of Oklahoma, Norman, in 1972 and 1975, respectively.

In 1975, he was appointed an Associate Professor, and in 1978, as a Professor and Chairman of the Department of Control Engineering, NCTU. In 1981, he became a Professor and Director of the Institute of Control Engineering, NCTU. In 1986, he was a Visiting Professor and, in 1987, a Full Professor of electrical engineering with the University of Kentucky, Lexington. In 1990, he was a Professor and Chairman of the Department of Electrical Engineering, National Taiwan University of Science and Technology (NTUST), Taipei. In 1998, he became the Professor and Dean of the Office of Research and Development, NTUST. In 2000, he was appointed Chair Professor with the Department of Electrical and Control Engineering, NCTU. Since 2004, he has been with the National Taipei University of Technology (NTUT), Taipei, where he is currently the President.

Prof. Lee received the Distinguished Research Award from the National Science Council, R.O.C., in 1991-1998; the Academic Achievement Award in Engineering and Applied Science from the Ministry of Education, R.O.C., in 1997; the National Endow Chair from the Ministry of Education, R.O.C., in 2003 and 2006, respectively; and the TECO Science and Technology Award from the TECO Technology Foundation in 2003. He was elected as a Fellow of the IEE in 2000. He became a Fellow of New York Academy of Sciences in 2002. He has served as a member of the Technical Program Committee and a member of the Advisory Committee for many IEEE-sponsored international conferences. He is currently the Vice President for the IEEE Conference of the Systems, Man, and Cybernetics Society. 